\title{
TEXTOS MÉDICOS EXTRAACADÉMICOS: DIFUSIÓN DE PRONÓSTICOS, RECETARIOS, HERBARIOS Y TRATADOS DE ALIMENTOS MEDIEVALES EN ROMANCE PENINSULAR
}

\author{
MEDICAL EXTRAACADEMIC TEXTS: SPREADING OF \\ PROGNOSTICS, RECIPES, HERBALS AND TREATISE OF \\ FOOD IN LATE MEDIEVAL PENINSULAR ROMANCE
}

\section{Jesús PENSADO FIGUEIRAS}

Universidade da Coruña

jesus.pensado@udc.es

\begin{abstract}
Resumen: Se consideran textos de medicina extraacadémicos aquellos tratados de carácter divulgativo que exponen de forma sucinta la teoría básica y la praxis indispensable para el ejercicio de la medicina. Estas obras se reunían en compilaciones y procuraban facilitar la labor de médicos, boticarios, barberos y cirujanos alejados de los centros de estudios medievales y con poco conocimiento del latín. Su propia naturaleza favoreció una importante difusión manuscrita, que ha sido objeto de escasa atención por parte de los estudios filológicos modernos. Sin embargo, basta con rastrear sus precedentes cultos para comprobar que la composición de estas obras parte de textos relevantes de la medicina greco-latina y árabe y que su carácter divulgativo no es sinónimo de falta de rigor científico. El presente artículo anali-
\end{abstract}


za el recorrido de algunos de estos textos de medicina práctica, desde las posibles fuentes hasta su traducción al romance y su circulación por los distintos reinos peninsulares de la baja Edad Media.

Abstract: It is considered as an extraacademic text about medicine a spreading treatise that expounds in a succinct way the basic theory and the essential praxis for the practice of medicine. These works were gathered in compilations and attempted to make easier the work of doctors, pharmacists, barbers and surgeons working away from medical centres and very small knowledge of Latin. Their own nature helped an important handwritten diffusion, which has been an object of very little attention by modern philological studies. However, it is enough to trace their cultural precedents to prove the composition of these studies which comes from outstanding texts of GrecoLatin and Arabian medicine, and their spreading condition does not mean absence of scientific rigor. This article analyses the trajectory of some of these texts on practical medicine from their possible sources to their translation into Romance and their spreading across the different peninsular kingdoms during the late medieval period.

Palabras clave: Difusión textual. Pronósticos. Herbarios. Recetarios. Tratados de alimentos.

Key words: Textual spreading. Prognostics. Herbals. Recipes. Treatises of food.

\section{INTRODUCCIÓN}

El estudio de los textos médicos castellanos de la época bajomedieval y proto-renacentista, comprendido como disciplina filológica sólida y asentada en el tiempo, comenzó a realizarse hacia el último tercio del siglo $\mathrm{XX}^{1}$ y vino a completar ciertos esfuerzos aislados de edición textual realizados con anterioridad $^{2}$. En aquel momento, los tratados médicos castellanos recibieron una atención renovada, comenzando a ser transcritos por el Seminario

\footnotetext{
1 No cabe duda de que estos estudios no existirían sin la labor fundamental realizada por unos cuantos investigadores a partir de los años 50 del siglo pasado. De los diversos nombres que cabría citar, sobresalen sin duda la impecable labor de catalogación de los textos médicos realizada por Guy Beaujouan (1972) y su análisis historiográfico efectuado por Luis García Ballester (2001). De cada autor solo cito la obra que condensa el trabajo realizado, aunque el número y la influencia de su obra respectiva es mucho mayor.

2 La edición del Tratado de las fiebres, de Isaac Israelí, llevada a cabo por el padre Llamas (Israelí, 1945), es ejemplo de ello.
} 
Hispánico de Estudios Medievales en la universidad de Wisconsin-Madison, llegando a los 55 textos repertoriados en la actualidad. La conocida finalidad de informatización del proyecto de Madison terminó creando una importante base de datos, de gran ayuda para el estudio de este tipo de textos en castellano. A partir de aquí, comenzaron a realizarse ediciones críticas en las que se detectaba ya una mayor profundidad en el estudio de los textos médicos medievales, como las ediciones del Menor daño de la medicina de Alfonso Chirino y del Compendio de la humana salud de Johannes de Ketham, realizadas por M. ${ }^{a}$ Teresa Herrera (Ketham, 1973; 1990); o La Suma de la Flor de Cirugía de Fernando de Córdoba, llevada a cabo por Ignacio Pérez Pascual (Córdoba, 2002). Asimismo, estas ediciones se vieron acompañadas de un importante número de artículos sobre cuestiones médicas medievales, en sus aspectos fundamentalmente lexicográficos. Por su parte, los textos médicos en catalán también conocieron una atención significativa, sobre todo en la primera mitad del siglo XX con la labor de Josep Ribelles i Comín o Lluís Faraudo de Saint-Germain. Esta atención decaería posteriormente antes de retomar su actividad a comienzos del presente siglo, en particular gracias al trabajo del grupo que Lluis Cifuentes Comamala ha ido formando en torno al proyecto sciencia.cat.

No obstante, se observa que, tanto en castellano como en catalán, los textos editados tienen autores bien conocidos o sus fuentes latinas son fácilmente reconocibles, mientras que los tratados u opúsculos anónimos no gozaron de la misma consideración por parte de la crítica moderna. Estos textos se sitúan al extremo de las llamadas obras de carácter extraacadémico, consideradas como una literatura menor, de gran pobreza científica y con elementos supersticiosos, debida a autores anónimos que vinculaban sus escritos al nombre de un médico famoso (García Ballester, 2001: 270; Taavitsainen, 2004: 42). Para una mayor difusión, solían reunirse en códices, denominados mayoritariamente «compendios», dirigidos a practicantes de la medicina poco conocedores del latín, que pretenderían dar respuesta sencilla a las cuestiones fundamentales de teoría y praxis médica necesarias para curar a un paciente.

El presente artículo pretende trazar la difusión textual de algunos de estos textos extraacadémicos que gozaron de cierta consideración en la baja Edad Media, a juzgar por la relevancia de sus fuentes o por el número de copias que se ha ido detectando en el análisis filológico. Para ello, conviene llamar la atención sobre la necesidad de trabajar en paralelo con los tratados médicos medievales redactados en las diferentes lenguas de la Península, en particular el castellano y el catalán. En efecto, se comprueba que en un nú- 
mero significativo de casos, las diferentes versiones provienen de una traducción del latín a una lengua vulgar peninsular: a partir de esta versión primera, su difusión continuó en romance, traduciéndose a otras lenguas peninsulares y transmitiéndose de forma independiente por los diferentes reinos de la geografía española. De estos tratados, solo es posible conjeturar con fundamento una posible jerarquía de las distintas traducciones peninsulares en el herbario Macer Floridus. En lo que respecta a los demás textos, no he localizado pasajes que permitan dilucidar de forma concluyente que la versión castellana proceda de la catalana o viceversa, aunque no cabe duda de que las distintas copias provienen de una única traducción de una lengua culta. A partir de aquí, sus vicisitudes fueron diversas e independientes ${ }^{3}$.

\section{LOS TEXTOS EXTRAACADÉMICOS}

\subsection{El Macer Floridus}

No todos los tratados médicos de carácter divulgativo eran anónimos. Algunos contaban con atribuciones autoriales más o menos contrastadas y aceptadas por la tradición. Uno de los más difundidos en la Edad Media, tanto en latín como en las lenguas vulgares fue el herbario De viribus herbarum, también conocido como Macer Floridus, atribuido a Odo Magdunensis. La fuente latina cuenta además con la edición crítica de Ludwig Choulant (1832), casi bicentenaria, pero realizada con gran criterio filológico.

Este herbario tiene la peculiaridad de ser el único tratado médico que presenta tres versiones bajomedievales en romance peninsular, en castellano, catalán y aragonés. Solo una de ellas proviene del latín, mientras que las demás son traducciones realizadas a partir de esa primera versión romance. El número de copias conservadas indica que la difusión de este herbario en España fue tan importante como en el resto de Europa, al traducirse, solo hasta el siglo XVI, al alemán, al francés, al danés, al italiano, al holandés y

3 Con el fin de mantener una cierta coherencia en un artículo de carácter fundamentalmente divulgativo, limitaré mi exposición a la difusión de ciertas obras incluidas en el códice Antiguo Medico de medicina medieval conservado en la biblioteca del palacio Zabálburu, de Madrid. Solo expondré los grandes rasgos de este compendio y citaré la bibliografía esencial para la buena comprensión de este artículo, sin extenderme en referencias que considero fuera de lugar. Para una argumentación precisa de las hipótesis expuestas, bibliografía y transcripción de la mayoría de estos tratados, remito al lector a mi tesis doctoral (Pensado Figueiras, 2013). Me limitaré a precisar cada manuscrito en aquellos lugares del artículo en que sea imprescindible. Para la localización precisa de cada obra, con indicación de manuscrito y folio, cf. el Apéndice. 
al inglés (Capuano, 1991: 182). De la versión aragonesa da noticia Guido Mensching (2002) y solo se conoce una copia, que aún permanece inédita. La versión catalana, que fue la primera en contar con edición crítica moderna, realizada por Faraudo de Saint-Germain (1955-1956), se conserva en tres manuscritos ${ }^{4}$ y su texto está prácticamente completo. Los testimonios castellanos son todos parciales; el más cercano a la disposición latina de plantas es el que figura en el códice Antiguo Medico de la Biblioteca del palacio Zabálburu, de Madrid. Pero su texto se trunca en la descripción del «Elleborus Albus», por lo que no se recogen más que 59 de las 77 plantas que conforman el corpus tradicional del De Viribus Herbarum latino. El testimonio de la Biblioteca Colombina ${ }^{5}$ presenta también un número significativo de plantas, si bien estas ya no se disponen en el orden latino tradicional, sino por orden alfabético y con capítulos interpolados que no eran traducción del De Viribus Herbarum latino. Los demás testimonios son mucho más breves, pero parecen provenir de la misma traducción castellana que los dos ya referidos. La primera de estas copias breves presenta seis capítulos del herbario en el manuscrito II-3063 de la Real Biblioteca, que ya había sido transcrita por el equipo de Madison, pero no individualizada, al encontrarse interpolada en un breve recetario para diversas afecciones. El segundo, también parcial, se encuentra en el manuscrito 2328 de la Biblioteca Nacional, códice inédito del siglo XVI y al que se ha dedicado poca atención, aunque parece presentar textos muy interesantes de medicina y fitoterapia. En un amplio compendio que mezcla recetas y virtudes de plantas, aparecen interpolados y sin orden aparente cuatro capítulos del Macer Floridus castellano, además de una serie de recetas con el mismo origen bajo el epígrafe «Medicamentos de Maçer». Sin embargo, la traducción de este capítulo es independiente de la que aquí se analiza, con virtudes del herbario y otras recetas de origen diverso, todo ello extractado en gran parte del Thesaurus Pauperum. Es precisamente en la traducción castellana de este recetario latino, el Thesoro de los pobres, donde se localiza una brevísima descripción de cuatro virtudes de la «Artemisia», recogidas bajo tres epígrafes en uno de sus capítulos finales. Al tratarse de un texto muy breve, es aventurado conjeturar que estos pasajes provengan de la misma traducción del Macer Floridus castellano que los demás testimonios, aunque tal

4 Cf. Apéndice. Para completar la transmisión catalana de este herbario conviene consultar el artículo de Laura Ramello (1994), que descubre y analiza una importante interpolación del Macer Floridus, también en catalán, pero a partir de una traducción independiente de la editada por Faraudo de Saint-Germain.

5 Biblioteca Colombina ms. 7-6-27-1 -transcrito por Conerly, Adermagni y Richards (1986)-. 
conjetura no debe descartarse por el momento. Por último, Richard Kinkade (1971) transcribe en nota unas líneas de este herbario localizadas en el manuscrito 3369 de la Biblioteca Nacional de España, aunque no identifica correctamente a qué obra pertenecen ${ }^{6}$.

A pesar de no contar con una copia castellana completa, el análisis textual ha demostrado que el texto catalán proviene del castellano. Mensching (2002) aventura incluso que el aragonés podría haber sido la lengua primera a la que se tradujo el texto latino. En cualquier caso, el Macer Floridus es un claro ejemplo de la vitalidad de los textos médicos. En la baja Edad Media los fines prácticos de curación de los enfermos no conocían barreras lingüísticas. Los textos médicos de los diferentes reinos se transmitían, copiaban y traducían indistintamente de una lengua a otra con la única intención de difundir el conocimiento científico, por lo que su estudio en paralelo es indispensable para obtener una visión cabal de estos textos. No obstante, aunque interesante para conocer la circulación escrita en los centros de saber, estos estudios contrastivos no deben limitarse a una mera competición interlingüística para discernir cuál fue la primera traducción al latín. Más importante que ello, el análisis conjunto es esencial para fijar los textos en ambos idiomas, para conocer qué familias de manuscritos latinos circularon y fueron traducidas y para comprender, en definitiva, por qué vías concretas se difundían los conocimientos técnicos en la Península Ibérica.

\section{TEXTOS ANÓNIMOS}

\subsection{Pronósticos pseudo-hipocráticos}

La versatilidad de los textos médicos medievales se vuelve más evidente en aquellos tratados que figuran sin autoría expresa. La composición de la mayoría de ellos parte de unas premisas constantes, al tomar apoyo generalmente en la enorme autoridad de los grandes divulgadores de la medicina greco-latina y árabe. En ocasiones, sus fuentes últimas son fácilmente reconocibles, al tomar a menudo la base textual de Hipócrates, Galeno o Dioscórides para su composición, enriquecida con posteriores aportaciones de la tradición médica. Ejemplo de ello es la versión en romance peninsular de los pronósticos pseudo-hipocráticos, tratados que procuraban deducir la enfermedad o el tiempo de vida que le restaba al paciente a partir de los síntomas que este manifestaba. La colación de

6 Kinkade (1971: 261-262, nota 2). 
los textos en vulgar peninsular con las respectivas ediciones latinas y con las copias en occitano permite trazar la difusión de estos textos por el sudoeste de Europa y comprobar que existía una comunicación fluida en la transmisión de los estudios de medicina en la época bajomedieval.

El gran trabajo realizado por Rosa Kuhne Brabant (1987-88, 1989, 1990) en la recopilación, estudio, edición y traducción de la versión árabe que dio lugar a la llamada segunda recensión de estos pronósticos ${ }^{7}$, conocida como Capsula eburnea, permite ir más allá del latín en el estudio de las familias de la transmisión textual de las que surgieron los textos en romance. Por ejemplo, el número variable de pronósticos en árabe, que parte de 25 para superar las 30 prenociones en algunos testimonios, queda fijado en 24 en la traducción al latín debido a la fusión de dos pronósticos árabes. Las copias sucesivas mantendrían este número, que continuaría en la traducción castellana. También es significativa la primera recensión llamada Prognostica Democriti, que no conoce versión árabe. En ella se observa que los pronósticos 13 y 14 de la versión latina se fusionaron asimismo en uno solo en las tres versiones identificadas en castellano, en la versión catalana de la Biblioteca Colombina y en la traducción normanda conservada en un manuscrito del Trinity College conocido como Trinity Practica (Hunt, 1997). Aunque la traducción normanda no parece presentar relación directa con la castellana o la catalana, tal coincidencia en ámbitos geográficos relativamente alejados indica que la línea de transmisión peninsular y la normanda partieron de copias latinas cercanas y confirma la fluidez ya expresada de la circulación de textos médicos en la Edad Media. En lo que respecta a este tratado, llama poderosamente la atención la relación existente entre una variante castellana ${ }^{8}$ y la versión catalana. Los vínculos que se establecen entre ellas y con los demás testimonios inducen incluso a sospechar que el copista de este texto castellano pudo haber tenido en su poder ambos testimonios en romance y acaso también el latino para componer esta nueva versión ${ }^{9}$. La multiplicidad de

7 Sudhoff (1915-1916).

8 Versión parcial del ms. 2262 de la Universidad de Salamanca.

9 El siguiente pasaje puede servir de ejemplo de la naturaleza compleja de la versión parcial del ms. 2262 de la Universidad de Salamanca:

\begin{tabular}{|c|c|c|c|c|}
\hline Texto latino & Zabálburu & Sal. 2262 & $\begin{array}{c}\text { Sal. } 2262 \\
\text { (versión parcial) }\end{array}$ & Bib. Col. 7-4-27 \\
\hline $\begin{array}{l}\text { Item qui una in causa } \\
\text { fuerit, si sub lingua } \\
\text { illi papula apparuerit } \\
\text { sicut lenticula. }\end{array}$ & $\begin{array}{l}\text { E si el enfermo } \\
\text { oviere la barvilla o } \\
\text { la raniella quebrada, } \\
\text { si le fallares un grano } \\
\text { tamaño como una } \\
\text { lanteja. }\end{array}$ & $\begin{array}{l}\text { E si el enfermo } \\
\text { ouiere la baruiella o } \\
\text { reniella quebrada si } \\
\text { le fallares vn grano } \\
\text { tamaño commo vna } \\
\text { lenteja. }\end{array}$ & $\begin{array}{l}\text { Si el enfermo ouiere } \\
\text { deyuso de la lengua } \\
\text { ranilla quebrada } \\
\text { catale si fallares vn } \\
\text { grano (^asi) çerca } \\
\text { asi commo lenteja. }\end{array}$ & $\begin{array}{l}\text { Item, si lo malalt aura } \\
\text { deius la lengua talla- } \\
\text { dura ho trencadura, } \\
\text { guarda si hi trobaras } \\
\text {.i. granell tan gran } \\
\text { com .i. lentilla. }\end{array}$ \\
\hline
\end{tabular}


relaciones establecidas pone de manifiesto la complejidad de la red de transmisiones que tales textos pueden llegar a establecer en su intrincado proceso de difusión.

La labor de identificación de fuentes de los tratados de prognosis es ardua, y más aún para aquellos de carácter general en los que debemos contentarnos con afirmar que la fuente primera es Hipócrates. En efecto, en su mayoría solo cabe reconocer una total incapacidad para encontrar textos más directos de los que surgirían las versiones en vulgar peninsular. Es lo que sucede en aquellas series de pronósticos denominadas «de origen incierto», textos que mezclan prenociones con base hipocrática y experimentos basados en la fitoterapia y en la zooterapia, cuya tradición directa, latina o árabe, no ha podido ser identificada.

A pesar de ello, es posible localizar concomitancias con algunas series catalogadas y estudiadas. Es el caso de los Indicia Valetudinum (Kühlewein, 1884; Avagliano, 1994). Es esta una serie concreta que no deduce el tiempo de vida que le resta al paciente - como es el caso de los Prognostica Democriti o la Capsula eburnea - sino que identifica la enfermedad que sufre el enfermo a partir de ciertos síntomas, la mayoría de ellos de carácter visible para el médico, aunque no todos. Otra serie de prenociones, los llamados Prognostica Galieni (Nutton, 1970), figura también de forma parcial, interpolada entre los pronósticos de origen incierto en los testimonios en vulgar peninsular. Su estructura conforma un brevísimo texto del que existen numerosas versiones que solo tienen en común la famosa descripción de la facies hippocratica y la relación del enfermo con el sueño. Tanto los Indicia Valetudinum como los Prognostica Galieni pudieron haber sido extractados del texto original en algún momento de la difusión textual, probablemente en latín, para transmitirse posteriormente junto con otros pronósticos de origen diferente antes de su traducción al romance. Incluso cabría la posibilidad de que este proceso continuara en lengua vulgar. La escasez de manuscritos impide afirmarlo con seguridad, aunque la exposición precedente de las interpolaciones del Macer Floridus castellano en diversos recetarios parece ser indicio de que los textos no quedaban fijados tras su traducción a las lenguas vulgares, sino que los copistas seguían extractándolos y refundiéndolos en copias sucesivas. Veremos otros ejemplos más adelante.

Esta hipótesis de reelaboración continua de los textos médicos dificulta el rastreo de sus precedentes y fuentes. Pero un análisis en profundidad permite indicar que el carácter extraacadémico de estos tratados no implica que su composición no se haya realizado acudiendo a textos fiables y de recono- 
cido prestigio. Continuando con los pronósticos denominados «de origen incierto», se localizan paralelismos con pasajes del Tratado de las fiebres de Isaac Israelí (1945; 1984), concretamente en párrafos del epígrafe «De las señales que anunçian las dolençias». Sin embargo, la mayoría de estos párrafos presenta una divergencia fundamental, ya que este capítulo de Isaac Israelí relaciona constantemente los signos anunciatorios con la fiebre, mientras que en las versiones en romance su mención es ocasional. La propia estructura de los pasajes cotejados parece dejar claro que no existe relación textual directa entre el tratado de Isaac Israelí y los pronósticos que nos ocupan. Es más probable que el autor hebreo hubiese utilizado la obra hipocrática o alguna composición posterior con base en ella para el capítulo cuarto de su tratado, coincidiendo así, de forma episódica, con el texto castellano. Pero tales coincidencias parecen poner de relieve que este tipo de tratados de origen hipocrático o pseudo-hipocrático gozaron de gran popularidad y difusión, hasta el punto de que se encuentran redacciones independientes del mismo pronóstico en obras de distinta lengua y procedencia.

Algunas de estas prenociones presentan, asimismo, ciertos paralelismos con pasajes en árabe de la misma naturaleza, aunque su relación no es lo bastante sólida para aventurar que se trata de una fuente de los pronósticos castellanos. Kuhne Brabant (1990b) edita estos textos, localizados en la obra Fî l-mawt al-sarî, además de algunos pronósticos localizados en el Kitab al-dury que ya no figuran en la traducción latina de la Capsula eburnea (Kuhne Brabant, 1990). Las semejanzas son, insisto, lejanas y no es adecuado hablar de fuentes sino de precedentes o textos paralelos, aunque existen semejanzas significativas entre algunos pasajes en castellano y en árabe ${ }^{10}$. Tal afinidad podría inducir a la hipótesis de que existieron nuevas relaciones

10 Basten tres ejemplos para mostrar sus similitudes:

\begin{tabular}{|c|l|}
\hline \multicolumn{1}{|c|}{ Códice Zabálburu } & \multicolumn{1}{c|}{ Kitab al-dury } \\
\hline $\begin{array}{l}\text { 37. Nonbrar los muertos en las enfermedades } \\
\text { agudas es señal de muerte. }\end{array}$ & $\begin{array}{l}\text { Códice Zabálburu Cuando veas al enfermo desvariar } \\
\text { nombrando a los muertos [...] eso es indicio de que } \\
\text { la muerte se producirá rápidamente. }\end{array}$ \\
\hline $\begin{array}{l}\text { 39. Grante set e sudor frio en las fiebres agudas } \\
\text { es señal de muerte. }\end{array}$ & $\begin{array}{l}\text { Fîl-mawt al-sarî } \\
\text { 1. El sudor frío en las fiebres agudas es signo de } \\
\text { muerte. }\end{array}$ \\
\hline $\begin{array}{l}\text { 43. Quando viniere al enfermo espasmo, que es } \\
\text { falleçimiento de rosollo, sobre la fiebre aguda, es } \\
\text { señal de muerte. }\end{array}$ & $\begin{array}{l}\text { 2. Si una fiebre se apodera de alguien y a este } \\
\text { se le agita el corazón y le dan palpitaciones y, al } \\
\text { mismo tiempo, le sobrevienen hipo y dificultad } \\
\text { para respirar y siente presión en el ombligo, } \\
\text { morirá a las diez horas de padecer esta dolencia. }\end{array}$ \\
\hline
\end{tabular}


de pronósticos, acaso escritas en original árabe, a juzgar por la cercanía de la versión castellana con los textos editados por Rosa Kuhne; o bien en hebreo, si se tienen en cuenta los paralelismos con la obra de Isaac Israelí. En cualquier caso, son textos de cierta amplitud, coherentes y de origen distinto a las recensiones ya aludidas de los Prognostica Democriti y de la Capsula eburnea.

Volviendo a la tradición latina, conviene destacar la relación paralela, aunque de nuevo indirecta, entre las versiones en romance y una parte de los versos espurios ${ }^{11}$ de la obra Flos Medicine. Este testimonio latino, cuya fuente aún no ha sido identificada, constituye una redacción diferente de pronósticos que ya circulaban en otras recopilaciones y en distintos idiomas. Toda esta confluencia de redacciones indica que las versiones en romance pudieron haber sido compuestas a partir de tratados independientes de naturaleza semejante, reunidos de forma desordenada, con el objetivo de deducir el tiempo restante de vida a partir del aspecto y de las reacciones físicas del paciente. Su difusión por la Península sigue las mismas pautas que se han observado en los tratados anteriores, con una misma traducción, acaso del latín al vulgar, y una posterior versión a la otra lengua romance conservada. En este caso, los pronósticos de origen incierto pueden dividirse en dos series distintas. De la primera de ellas se conservan cuatro testimonios en romance, tres de ellos en castellano y uno en catalán. Pero no hay indicios suficientes para argumentar una jerarquía en estas traducciones medievales, pues las versiones conservadas presentan todas ellas deturpaciones y características propias que impiden establecer hipotéticas subordinaciones textuales. De la segunda serie solo se conservan versiones castellanas, aunque no se debe descartar la aparición de nuevas copias, en catalán o en otra lengua peninsular, que enriquezcan la transmisión romance de estos tratados.

\subsection{Pronósticos por el pulso y por la orina}

Un caso particularmente complejo de difusión del saber y de su plasmación por escrito lo ofrecen los pronósticos por el pulso y por la orina. Son tratados breves, pero de una compleja tradición, que dan buena cuenta de la

11 La edición de Flos Medicine (Frutos, 2010) recoge 1475 versos de los 2130 de Salvatore de Renzi en su primer tomo (el más cercano a la edición de Frutos) y de los 3520 en su tomo quinto (Renzi, 1852-1857). La editora argumenta que no forman parte del texto inicial de la obra latina: «Ambas versiones [...] se caracterizan por no tener en cuenta la transmisión del texto al incluir numerosísimos versos de otras fuentes que alejaron totalmente la Flos medicine del texto genuino que se extendió por toda Europa» (Frutos, 2010: 108). 
dificultad que puede entrañar el estudio de estos tratados, así como de la importancia de recorrer con calma y cautela el camino que recorrieron a lo largo de los siglos para evitar conclusiones precipitadas.

El tratado de los pronósticos por el pulso comienza exponiendo brevemente en sus primeros párrafos la teoría sobre la naturaleza de los latidos del corazón, su relación con los humores y su comportamiento en las distintas estaciones del año. Tras este calendario se inicia la prognosis propiamente dicha, realizada según las características del pulso captado al colocar los dedos sobre el corazón del paciente. Ordenados por el orden clásico de la cabeza a los pies, aunque no de forma estricta, cada pasaje pronostica una dolencia concreta según se perciba el latido con más fuerza en uno u otro dedo o falange.

Este texto, del que no se ha localizado fuente latina o precedente cercano, cuenta con cinco testimonios en castellano, dos de ellos parciales. De estas copias destaca la que aparece interpolada en la traducción castellana del Compendio de la humana salud de Johannes de Ketham (1990), que no tiene correlato alguno en la versión latina de este texto. El testimonio interpolado aparece modificado tanto sintáctica como lexicográficamente, aunque no hay duda de que se trata de una reelaboración de la misma traducción de la que surgieron las demás copias. Además de las castellanas, se conservan siete versiones catalanas; a pesar de que el texto de todas ellas es muy semejante, cinco se difunden de manera anónima y las dos restantes bajo la autoría de un importante médico catalán de la Edad Media llamado Antoni Ricart. No parece necesario incidir en la gran dificultad que supone establecer un stemma de estos textos, y mucho menos dilucidar sobre cuál fue la traducción primera de un texto latino aún no localizado.

Los llamados Pronósticos por la orina, que deducen el tipo de enfermedad del paciente según el resultado de la uroscopia, presentan una complejidad aún mayor. La nómina de manuscritos que recogen estas prenociones en romance peninsular es más importante que en el tratado por el pulso, con numerosas copias de este texto, la mayoría parciales y algunas muy breves. Solo dos están en castellano, mientras que las demás provienen de manuscritos en catalán. A pesar de este desequilibrio numérico, el análisis textual tampoco permite afirmar de forma definitiva que el catalán haya sido el idioma romance al que se tradujo la versión latina de este tratado. De hecho, la propia consideración de tratado es aquí discutible. Así como en los pronósticos por el pulso existe un incipit que lo distingue del texto anterior en las diferentes copias, esto no es unánime en los pronósticos por la orina, 
puesto que solo sucede en algunas versiones catalanas. El manuscrito salmantino 2262 presenta, por ejemplo, un incipit genérico para el pulso y la orina: «Tratado del conoçimiento de las orinas \& de los pulsos»; seguidamente comienza a exponer los pronósticos que derivan de la uroscopia, con la fórmula «e primeramente diremos de las orinas». En este códice figuran ocho folios dedicados a la orina, con una parte inicial teórica en la que se identifican diversos pasajes de autores de la escuela de Salerno, además de otros con fuentes aún no localizadas. Tras el epígrafe que expone la influencia del calendario y de los humores sobre la orina, comienza la pronosticación propiamente dicha, que se extiende a seis folios antes de que se inicie la parte común con los demás textos en romance. Algo semejante sucede con el manuscrito de la Biblioteca Colombina, que dedica cuatro folios a una posible traducción de una parte de las Regulae Urinarum de maestro Mauro (Renzi, 1854), antes de exponer la parte común con los demás textos catalanes y los castellanos.

Las fuentes latinas tampoco ayudan a esclarecer la transmisión de este opúsculo del latín al romance y su posterior difusión por la Península Ibérica. De hecho, los textos latinos localizados no son fuente directa de la versión romance para un tratado muy difundido y extractado ya en latín, con pasajes que se pueden rastrear en distintos manuscritos. Las dos versiones más cercanas a las castellanas ${ }^{12}$ permiten detectar lecturas erróneas en los textos castellanos, pero no bastan para resolver las numerosas cuestiones que surgen de la colación textual y que seguirán sin respuesta por el momento.

\subsection{El Libro de las cosas que el hombre come}

El texto que nos servirá de eje para observar la transmisión de los tratados de alimentos de la época medieval figura en el códice Zabálburu y recibe en su incipit el título Libro de las cosas que el hombre come. Esta obra describe las virtudes terapéuticas y nutritivas de 73 simples $^{13}$, en su mayoría vegetales, ordenados por categorías: pan, legumbres, verduras, frutas y derivados, carne, huevos, leche, manteca, queso y pescado.

Este tratado de los alimentos recoge información muy difundida en otras obras de alimentación conocidas en la Edad Media, tanto latinas como ára-

12 Wien. Cod. 2532. S. 59r-64v (Wentzlau, 1923).

13 Esta suele ser la denominación de los materiales de procedencia orgánica o inorgánica, que sirven por sí solos a la medicina o que entran en la composición de un medicamento. 
bes y castellanas ${ }^{14}$. Cabe destacar los numerosos paralelismos entre este texto y la obra castellana Sevillana medicina de Juan de Aviñón (1999); o los que se detectan con el tratado árabe de Ibn Habīb (1992), titulado Mujtasar fi l-tibb (Compendio de medicina). En efecto, casi la mitad del Libro de las cosas... encuentra elementos comunes en la parte dedicada a la alimentación de Ibn Habīb. Pero no parece probable que tales semejanzas indiquen una fuente directa, sino la existencia de fuentes comunes a ambas obras. De naturaleza semejante es la relación del Libro de las cosas... con el tratado Vergel de Sanidad, cronológicamente posterior, compuesto por Luis Lobera de Ávila (1542). El autor compuso en latín y castellano una amplia obra dedicada a los aspectos habituales del ejercicio, el baño, el sueño, el regimiento de caminantes y marinos y, por supuesto, las propiedades nutritivas y terapéuticas de los alimentos. Las autoridades estudiadas son las habituales, tomando todas ellas como fuente primera las obras que Galeno dedicó a los alimentos ${ }^{15}$. Por esta razón, no sorprende la presencia de abundantes paralelismos con los tratados posteriores, sin que de ello se pueda inferir la existencia de relación directa alguna.

La disposición de los simples en el Libro de las cosas... que he indicado más arriba también establece una relación significativa con textos castellanos de naturaleza similar, pues el orden de los alimentos fue experimentando una evolución progresiva a lo largo de los siglos. Como muestran Peña y Girón (2006), una buena parte de los textos árabes, latinos y castellanos, como el propio Vergel de Sanidad, siguen una disposición de alimentos que podría considerarse tradicional: pan, carne, huevos, leche y derivados, pescado, frutas y verduras. Por el contrario, tanto la obra del códice Zabálburu como otros textos castellanos ${ }^{16}$ alteran este orden y disponen los alimentos de origen animal ${ }^{17}$ tras los de origen vegetal, en concreto, después de las frutas y las verduras ${ }^{18}$. De esta manera, sería posible conjeturar que tal dis-

14 Basta consultar la obra de Peña y Girón (2006). Para una visión de conjunto de los Regimina Sanitatis, que incluían a menudo los tratados de alimentos, cf. el estudio de García Ballester et al. (1996).

15 Las obras de Galeno que se consideran fundacionales para los tratados de los alimentos son las siguientes: De alimentorum facultatibus (Kühn, VI, 453-748) y De simplicium medicamentorum temperamentis ac facultatibus (Kühn, XII, 1-377).

16 Es el caso de la Sevillana medicina (Aviñón, 1999) o el Libro de medecina llamado Macer (Maestro Gil, 1991).

17 En el tratado de los alimentos del códice Zabálburu esta separación se marca con un incipit: «Aqui comiença a demostrar de las carnes quales son cada una». No parece suficiente para considerarlo un tratado aparte, pues su texto coincide con el catalán, al igual que en los productos de origen vegetal.

18 El Menor daño de medicina (Chirino, 1973) sigue, en este sentido, un orden que le es propio. 
posición no es casual y acaso un estudio diacrónico de las variantes podría aportar resultados interesantes. Con los datos actuales no es posible dilucidar las razones de tal variación y su coincidencia en textos castellanos, que solo tienen en común el orden de los productos descritos. Además, la disposición canónica de los alimentos fue perdiendo importancia con el tiempo y ya se observa que el Liber de Conservanda Sanitate ${ }^{19}$ comienza su tratado por las carnes, continuando por los pescados, las legumbres y los huevos. En la época renacentista, los autores optaron por el orden alfabético, como es el caso del Regimen Sanitatis de Benedicto de Nursia ${ }^{20}$, abandonando la disposición de los alimentos según las categorías tradicionales del medievo.

Aunque de difusión mucho más sencilla que los pronósticos ya referidos, el tratado castellano también presenta correlato en catalán. La diferencia fundamental entre las versiones peninsulares radica en que el castellano está integrado en una descripción más amplia de cada simple y los pasajes comunes con el catalán se introducen en una segunda parte de cada descripción, mediante la fórmula introductoria «e dize otro sabio». En este tratado, el texto castellano es relativamente amplio, con una posible confluencia de varias tradiciones independientes. Por su parte, la versión catalana es mucho más breve y aparece tras el ya mencionado herbario Macer Floridus, sin incipit o marca que separe ambos tratados en dos de los testimonios conservados. A pesar de ello, el estudio textual demuestra que los pasajes comunes en castellano y catalán provienen, una vez más, de una única fuente. Más aún, al igual que se observaba en tratados anteriores, es casi seguro que una de las versiones en lengua vulgar proviene del otro texto romance. Cabría la posibilidad de conjeturar que el opúsculo catalán sería resultado de un extracto del tratado castellano, más amplio. Pero las deturpaciones existentes en el único testimonio castellano que ha llegado a nuestros días indican que tal conjetura parece poco verosímil. Por el contrario, este mismo argumento serviría para formular la hipótesis opuesta, esto es, una dependencia del $\mathrm{Li}$ bro de las cosas... con respecto a la versión catalana. En cualquier caso, ambos textos provienen de una misma traducción del latín, con difusión posterior en la Península Ibérica y traducción de una a otra lengua romance. Las únicas conclusiones que se pueden formular a este respecto indican que

19 García Ballester et al. (1996: 62). Es el caso del tratado de dietética de Jofroi de Waterford, editado por Schauweker (2007: 103 y ss.). Considerando que el capítulo sobre el vino y sus cualidades parece constituir un tratado aparte, la obra de dietética propiamente dicha comienza en el folio 114vb, describiendo las propiedades de las distintas carnes, leche y derivados, aves y huevos, pescado, cereales, legumbres, frutas, miel y especias. Termina con consideraciones sobre el baño y la sangría.

20 García Ballester et al. (1996: 92). 
la fuente última, aunque no directa, es Galeno y que la versión en catalán permite corregir ciertas lagunas y deturpaciones del texto castellano. De nuevo, la falta de documentos impide ir más allá en las conjeturas formuladas, aunque se vuelve a confirmar que los textos no quedaban fijados tras su traducción al romance, sino que el proceso de extracción y fusión, común en las versiones latinas, continuaba tras el paso al romance, siendo muestra de la gran vitalidad de la literatura médica en la baja Edad Media y en el protoRenacimiento.

\subsection{Los recetarios medievales}

Los recetarios representan probablemente el paradigma de la continua reelaboración textual expuesta en el presente artículo. La naturaleza de las recetas terapéuticas invita a ello, pues es habitual localizar prescripciones para una misma afección que parten de un simple o simples básicos, evolucionando posteriormente hasta alcanzar en ocasiones considerables grados de complejidad. El ejemplo más conocido y estudiado es el de la triaca, cuyo propio significado fue variando desde un inicial remedio contra venenos y mordeduras ponzoñosas a un genérico para enfermedades diversas. Su composición también sufrió transformaciones importantes, conociéndose triacas formadas por un único simple, el ajo, denominado «triaca agreste» por Galeno en el libro XII del De methodo medendi ${ }^{21}$, hasta los más de sesenta elementos que componen la llamada «triaca magna» ${ }^{22}$. En cualquier caso, un recetario suele estar compuesto a partir de extractos de otros textos, reunidos siguiendo alguna motivación no expresa por parte del copista. Tal modus operandi, habitual en los textos médicos, dificulta sobremanera no solo la localización de sus fuentes sino también el rastreo de su difusión textual.

En el caso del recetario del códice Zabálburu se observa, por ejemplo, que no presenta ninguna prescripción en común con el manuscrito salmantino 2262, con el que comparte bastantes tratados ${ }^{23}$. Solo se localiza un número significativo de recetas comunes en manuscritos en catalán, y de nuevo en una posible transmisión a partir de una misma traducción del latín a una lengua romance peninsular. El recetario de Zabálburu recoge 256 prescripciones, la mayoría de ellas en castellano, y algunas, muy pocas, en latín. De

21 Kühn (tomo X: 866).

22 El lector interesado puede consultar las consideraciones morales de la «triaca magna» realizadas por Domingo Guillén (1724).

23 Cf. Apéndice. 
nuevo, el ya mencionado manuscrito en catalán de la Biblioteca Colombina $^{24}$ comparte 178 recetas con el de Zabálburu, si bien hay indicios de probables lagunas del texto castellano en algunos pasajes presentes en el recetario catalán. Por lo tanto, es posible conjeturar que existió un tratado de al menos 178 recetas que se tradujo del latín al romance, aunque no hay indicios suficientes para concluir cuál fue el idioma de su traducción original en vulgar. A partir de esa primera versión romance, el texto se difundió por la Península Ibérica, apareciendo integrado en otros recetarios y traduciéndose al menos a otra lengua vulgar. O bien, en caso de que la traducción inicial fuese más amplia, extractado posteriormente e incluido en recetarios de diversa procedencia.

El estudio contrastivo permite localizar pasajes paralelos de estos recetarios en diversos textos tanto castellanos como catalanes, que indican relaciones esporádicas sin ninguna sistematización. La estructura de las recetas señala que tal concurrencia parece ser resultado del uso de fuentes latinas comunes y no de una difusión de las recetas tras su traducción al romance. Por ejemplo, se identifican recetas paralelas en 17 de los textos transcritos por Madison con un número que oscila entre una y siete prescripciones comunes con el texto de Zabálburu. Lo mismo sucede con los manuscritos catalanes, además de los ya mencionados, con recetas que comparten fuentes comunes ${ }^{25}$, en concreto, el célebre Thesaurus Pauperum (Da Rocha, 1972). Este recetario, atribuido al portugués Pedro Hispano -más tarde papa Juan XXI-, es a su vez una recopilación de recetas de diversas procedencias, que presenta la peculiaridad de citar la fuente de cada prescripción. Su enorme difusión lo convirtió en uno de los recetarios más influyentes de la tradición médica bajomedieval; su influjo perduró durante varios siglos, llegándose a localizar ciertos pasajes en obras publicadas en el Nuevo Mundo, como es el caso del Tesoro de medicinas para todas enfermedades de Gregorio López ${ }^{26}$. Solo en la Península han sido identificadas una versión catalana ${ }^{27}$ y cuatro castellanas, tres de ellas independientes: la primera es la traducción impresa en 1519, titulada Thesoro de los pobres en medicina et cirurgia, junto con su variante aljamiada, más breve, que se divulgó de for-

24 Ms. 7-4-27. Por su parte, el manuscrito 216 de la Universidad de Valencia recoge 93 recetas en común con el códice Zabálburu, también redactadas en catalán y todas presentes en el códice sevillano.

25 Es el caso de El receptari del sabadellenc Joan Martina (Perarnau i Espelt, 1992), el Mals $i$ remeis (Gómez i Gabernet, 1918) o el Micer Johan (Moliné i Brasés, 1913; Escudero, 1993).

26 Cf., por ejemplo, Guerra (1982: 252, líneas 19-20), en el apartado de la «hidropesia».

27 Tresor de pobres (Hispà, 1892). Aunque no he realizado un análisis exhaustivo, parece ser una traducción independiente de las versiones castellanas. 
ma manuscrita (Hespano, 1999). La tercera está constituida por buena parte del texto del manuscrito II-3063 de la Real Biblioteca, transcrita por el equipo de Madison con la abreviatura GIL y el comienzo «yncipid liber recebtarum Gilbertus», lo que indujo a considerarlo hasta hace poco un recetario atribuido a Gilberto Ánglico (Gilberto, 2002; García Ballester, 2001: 408) ${ }^{28}$. La cuarta versión, mucho más breve, también figura en este mismo manuscrito de la Real Biblioteca y es parte de la transcripción de Madison con la abreviatura SDM (Enriquez, 1992) ${ }^{29}$, conjunto de textos atribuidos a Juan Enriquez, entre los que figuran los ya mencionados capítulos del Macer Floridus.

Pero no es este el único tratado latino que aporta pasajes a las variantes castellana y catalana del recetario que nos ocupa, entre las consultadas. También es posible rastrear coincidencias con obras menos conocidas pero también influyentes, como la Tereoperica, compilación medicinal que presenta la descripción de la enfermedad, sus causas y las prescripciones pertinentes para curarla. Asimismo, se identifican otros tratados de mayor difusión y gran tradición en castellano, como el herbario de pseudo-Apuleyo, el Trotula o la Carta de Hipócrates a César. Por último, es digno de reseña otro texto menos conocido, que alterna pasajes en latín y romance normando, editado a partir del ya mencionado Trinity Practica y que también presenta textos paralelos con el recetario que nos ocupa.

\section{CONCLUSIÓN}

Los precedentes aquí expuestos muestran bien la variedad de fuentes utilizadas por los copistas para la composición de simples recetarios, destinados a un uso práctico. Esta misma riqueza documental, observada también en el análisis de pronósticos, herbarios o tratados de alimentos expuestos anteriormente, parece indicar que el carácter extraacadémico y esencialmente empírico de estas obras no excluía una composición rigurosa, basada en las autoridades en medicina griega, árabe y latina. La difusión posterior debió de ser amplia, con muchas más copias de las que se han conservado, a juzgar por el tipo de deturpaciones que se detectan en el aná-

28 Aunque hemos llegado a la misma conclusión de forma independiente, el honor de haber publicado primero esta identificación de GIL como traducción del Thesaurus Pauperum corresponde a Moral de Calatrava (2012: 362): «Por sus similitudes en estructura y contenido, es una traducción del Thesaurus pauperum de Pedro Hispano».

29 Esta identificación ya fue indicada en su momento por M. Lenkiewicz (1983: xxxi, 1xx y 1xxi). 
lisis textual. Estos mismos errores e importantes lagunas dan fe de la vitalidad de unos textos copiados con rapidez por considerarse útiles para la práctica de la medicina.

Cabe lamentar, de acuerdo con Iolanda Ventura (2011: 30-31), que las traducciones vernaculares solo jueguen un papel marginal en el estudio de la complejísima tradición manuscrita de las obras de medicina salernitana - y en ocasiones presalernitana-, limitándose en muchas ocasiones a una simple mención en la «lista de testimonios manuscritos». Sin embargo, se ha demostrado que los textos de medicina en vulgar revelan la existencia de familias de la difusión latina que acaso no se hayan conservado en la actualidad. Es el caso del Macer Floridus, el único texto de los analizados con un cotejo de fuentes exhaustivo, reflejado en el aparato crítico de Ludwig Choulant (1832). La fuente latina de la traducción peninsular del herbario no proviene de ninguno de los veintiún testimonios, manuscritos e impresos, colacionados por el estudioso alemán. Sus particularidades se detectan en códices diferentes, pero ninguna de las tradiciones textuales latinas presenta todas las variantes recogidas por los textos peninsulares. En consecuencia, las versiones en vulgar del De Viribus Herbarum demuestran que hubo otra familia de la transmisión textual latina que circuló por la Península Ibérica y que aún no ha sido objeto de estudio.

Desde el punto de vista lexicográfico, los textos en romance son indispensables para comprender la formación del lenguaje científico en los idiomas peninsulares. La propia existencia de traducciones medievales indica una madurez incipiente de las lenguas vulgares, que permite reflejar los matices expresados en las lenguas cultas. Desde la simple copia de étimos latinos o la transliteración de términos técnicos griegos o árabes en los textos más primitivos hasta la sobresaliente precisión del Andrés Laguna traductor de Dioscórides, el camino recorrido es amplio. Los textos analizados presentan significativas glosas semánticas ${ }^{30}$ que indican una labor de indagación léxica, a veces aún balbuciente, por parte de quienes traducían y difundían los textos médicos. De ahí la necesidad de continuar realizando estudios y ediciones críticas fiables de estas obras, como se viene haciendo en las últimas décadas, para poner de relieve su indudable importancia filológica y su aportación a la historiografía médica de la baja Edad Media.

30 Sirvan de ejemplo las siguientes: «espasmo, que es falleçimiento de rosollo»; «el abrotanon, que es romero»; «morçieguillo, que es dicho salvosoriz»; «en el prepuçio, que quiere dezir en el capillo de la pixa»; «roar, que quiere dezir tanto como robarvo»; «gomito, que quiere dezir bosamiento»; «yteriçia, que quiere dezir mudamiento de color natural en non natural». 


\section{APÉNDICE. Relación de obras, manuscritos y folios en las distintas versiones en castellano y catalán}

\begin{tabular}{|c|c|c|}
\hline Tratado & Versión en castellano & Versión en catalán \\
\hline Macer Floridus 31 & $\begin{array}{l}\text { - Antiguo Medico, } \\
\text { Zabálburu, ff. 39rb-61vb. } \\
\text { - Bib. Colombina ms. 7-6-27- } \\
\text { 132. } \\
\text { - BNE, ms. 2328, ff. 78v-81r. } \\
\text { - BNE 3369, ff. 169v-170r. } \\
\text { - Real Biblioteca, ff. 13va-14va. } \\
\text { - Thesoro de los pobres, 1519, f. } \\
\text { 24v. }\end{array}$ & $\begin{array}{l}\text { - Bib. Colombina, ms. } \\
\text { 7-4-27, ff. 20rb-46vb. } \\
\text { - Bib. Univ. de } \\
\text { Valencia, ms. } 216 . \\
\text { - Bib. Nat. de France, } \\
\text { ms. esp. } 21033 \text {. }\end{array}$ \\
\hline $\begin{array}{l}\text { Pronósticos de origen } \\
\text { incierto }\end{array}$ & $\begin{array}{l}\text { - Antiguo Medico, Bib. } \\
\text { Zabálburu, ff. 6va-7rb y 14va-17ra. } \\
\text { - Univ. de Salamanca, ms. 2262, } \\
\text { ff. 26r-26v, 37r-37v y 38v-40v34. }\end{array}$ & $\begin{array}{l}\text { - Bib. Colombina, ms. } \\
\text { 7-4-27, ff. 19rb-19vb }\end{array}$ \\
\hline Prognostica Democriti & $\begin{array}{l}\text { - Antiguo Medico, Bib. } \\
\text { Zabálburu, ff. 5ra-6va. } \\
\text { — Univ. de Salamanca, ms. 2262, } \\
\text { ff. 24v-26v y 35v-37r. }\end{array}$ & $\begin{array}{l}\text { - Bib. Colombina, ms. } \\
\text { 7-4-27, ff. 17vb-19rb. }\end{array}$ \\
\hline Capsula Eburnea & $\begin{array}{l}\text { - Antiguo Medico, Bib. } \\
\text { Zabálburu, ff. 17ra-18vb. } \\
\text { - Univ. de Salamanca, ms. 2262, } \\
\text { ff. 40v-42r. }\end{array}$ & \\
\hline Prognostica Galieni & $\begin{array}{l}\text { - Antiguo Medico, } \\
\text { Zabálburu, ff. 7va-7vb. } \\
\text { - Univ. de Salamanca, ms. 2262, } \\
\text { ff. 27r y 37v-38r. }\end{array}$ & $\begin{array}{l}\text { - Bib. Colombina, } \\
\text { ms. 7-4-27, f. 19vb. }\end{array}$ \\
\hline Indicia Valetudinum & $\begin{array}{l}\text { - Antiguo Medico, } \\
\text { Zabálburu, ff. 5ra-7rb. } \\
\text { - Univ. de Salamanca, ms. 2262, } \\
\text { ff. 26v y } 37 \mathrm{v} \text {. }\end{array}$ & $\begin{array}{l}\text { - Bib. Colombina, ms. } \\
\text { 7-4-27, ff. 20ra-20rb. }\end{array}$ \\
\hline
\end{tabular}

${ }^{31}$ Ya he mencionado la versión en aragonés, con pequeños pasajes que se recogen en Mensching (2002).

32 Transcrito por Conerly, Adermagni y Richards (1986).

${ }^{33}$ Para las referencias a los mss. de la Bibliothèque Nationale de France y de la Universidad de Valencia, cf. Faraudo (1955-56).

${ }^{34}$ Debido a la complicada paginación de este manuscrito, sigo la indicada en la transcripción de M. ${ }^{a}$ del Carmen de la Cal (1987). 


\begin{tabular}{|c|c|c|}
\hline Tratado & Versión en castellano & Versión en catalán \\
\hline Pronósticos por el pulso & $\begin{array}{l}\text { - Antiguo Medico, Bib. } \\
\text { Zabálburu, ff. 7vb-9va. } \\
\text { - Univ. de Salamanca, ms. 2262, } \\
\text { ff. 21v-23v y 38r. } \\
\text { - Real Biblioteca, II-3063, ff. } \\
\text { 78rb-78va. } \\
\text { - Ketham (1990), ff. 33r-34r. }\end{array}$ & $\begin{array}{l}\text { - Bib. Colombina, ms. } \\
\text { 7-4-27, f. 16rb-17vb. } \\
\text { - Bib. Nat. de France, } \\
\text { ms. 508, ff. 11rb-12rb. } \\
\text { - Univ. de Barcelona, } \\
\text { ms. 239, f. 14r-14v. } \\
\text { - Bib. Vatinaca ms. } \\
\text { Lat 4797, ff. 55r-56v y } \\
\text { 65r-70r. }\end{array}$ \\
\hline Pronósticos por la orina & $\begin{array}{l}\text { - Antiguo Medico, Bib. } \\
\text { Zabálburu, ff. 9va-10va. } \\
\text { — Univ. de Salamanca, ms. 2262, } \\
\text { ff. 8v-21r- }\end{array}$ & $\begin{array}{l}\text { - Bib. Colombina, ms. } \\
\text { 7-4-27, ff. 11ra-15vb. } \\
\text { - Bib. Univ. de } \\
\text { Valencia, ms. 216, ff. } \\
\text { 22ra-22va. } \\
\text {-Bib. de Catalunya, ms. } \\
\text { 864, ff. 2va-3rb. } \\
\text { - Bib. Nat. de France, } \\
\text { ms. 508, ff. 10rb-11ra. } \\
\text { - Univ. de Barcelona, } \\
\text { ms. 239, f. 14r-14v. } \\
\text { - Bib. Vatinaca ms. } \\
\text { Lat 4797, ff. 52r54r y } \\
\text { 58v-62r. } \\
\text { - Bib. Jagiellońska } \\
\text { ms. Hisp Qu 62, ff. } \\
\text { 50r-50v y 58v-60v }\end{array}$ \\
\hline $\begin{array}{l}\text { Libro de las cosas que el } \\
\text { hombre come }\end{array}$ & $\begin{array}{l}\text { - Medico, } \\
\text { Zabtiguo Bib. } \\
\text { Zabarlbu, ff. 34va-39rb. }\end{array}$ & $\begin{array}{l}\text { - Bib. Colombina, ms. } \\
\text { 7-4-27, ff. 47ra-48va. } \\
\text { - Bib. Univ. } \\
\text { Valencia, ms. } 216 . \\
\text { - Bib. Nat. de France, } \\
\text { ms. esp. } 210^{35} \text {. }\end{array}$ \\
\hline Recetario & $\begin{array}{l}\text { - Antiguo } \text { Medico, } \\
\text { Zabálburu, ff. 19ra-34va y } 63 \mathrm{r}-65 \mathrm{v}\end{array}$ & $\begin{array}{l}\text { - Bib. Colombina, ms. } \\
\text { 7-4-27, ff. 48va-59vb. } \\
\text { - Bib. Univ. de } \\
\text { Valencia, ms. 216, ff. } \\
\text { 8ra-14rb. }\end{array}$ \\
\hline
\end{tabular}




\section{REFERENCIAS BIBLIOGRÁFICAS}

AVAGLIANO, F. (1994). «Codex Casinensis 69: Anonymi Varia Excerpta Medica (saec. IX)». American Journal of Nephrology 14, 494-495.

AVIÑÓN, J. (1999). Sevillana Medicina, José Mondéjar (ed.). Madrid: Arco/Libros.

BEAUJOUAN, G. (1972). «Manuscrits médicaux du Moyen Âge conservés en Espagne». Mélanges de la Casa de Velázquez 8, 161-221.

CAL, M. del C. (ed.) (1987). Recetas (Salamanca: Biblioteca Universitaria 2262). Madison: Hispanic Seminary of Medieval Studies.

CAPUANO, T. M. (1991). «Medieval Iberian Vernacular Versions of the Herbal Called Macer Floridus». Manuscripta 35, 182-202.

CHIRINO, A. (1973). Menor daño de la medicina, M. ${ }^{a}$ Teresa Herrera (ed.). Salamanca: Universidad de Salamanca.

CHOULANT, L. (ed.) (1832). Macer Floridus, De Viribus Herbarum, Ludwig Choulant (ed.). Leipzig: Leopold Voss.

CONERLY, P.; ARDEMAGNI, E. y RICHARDS, R. M. (eds.) (1986). Macer herbolario. Madison: Hispanic Seminary of Medieval Studies.

CÓRDOBA, F. (2002). La Suma de la flor de cirugía, José Ignacio Pérez Pascual (ed.). Noia: Toxosoutos.

DA ROCHA PEREIRA, M. ${ }^{a}$ H. (1972). Obras médicas de Pedro Hispano. Coimbra: Acta Universitatis Conimbrigensis.

ENRIQUEZ, J. (1992). Secretos de la medicina, Enrique Jiménez Ríos (ed.). Madison: Hispanic Seminary of Medieval Studies.

ESCUDERO MENDO, A. (1993). Manuscritos de la Biblioteca de Catalunya de interés para la farmacia y las ciencias médicas: en particular el «Tresor de pobres» (Tesis doctoral). Barcelona: Universitat de Barcelona.

FARAUDO DE SAINT GERMAIN, L. (1955-1956). «Una versió catalana del Libre de les herbes de Macer». Estudis Romànics 5, 1-54.

FRUTOS GONZÁLEZ, V. (ed.) (2010). Flos medicine. Valladolid: Secretariado de Publicaciones e Intercambio Editorial, Universidad de Valladolid.

GALENO, C. (1821-1833). Opera Omnia (I-XX), C. G. Kühn (ed.). Leipzig: Car. Cnoblochii.

GARCÍA BALLESTER, L.; GIL-STORES, P. y MCVAUGH, M. R. (ed.) (1996). Opera Médica Omnia 3. Regimen Sanitatis ad Regem Aragonum de Arnau de Vilanova (vol. X.1). Barcelona: Fundació Noguera, Universidad de Barcelona. 
GARCÍA BALLESTER, L. (2001). La búsqueda de la salud. Sanadores y enfermos en la España medieval. Barcelona: Ediciones Península.

GILBERTO (2002). El libro de recetas (Madrid, Real Biblioteca, sign. II3063), 1sabel Zurrón (ed.). Madison: Hispanic Seminary of Medieval Studies.

GÓMEZ I GABERNET, F. (1918). «Regirant papers vells: mals i remeis». Nuevo Ambiente, 140.

GUILLÉN, D. (1724). Triaca Magna de los antiguos. Zaragoza: Pasqual Bueno.

HISPÀ, P. (1892). Tresor de pobres compilat per Pere Hispano (Papa Joan XXI), versió catalana del segle XIV, Jaume Collell i Bancells (ed.). Barcelona: Impr. Fidel Giró.

HISPANO, P. (1999). Tesoro de los proves: versão em judeu-castelhano aljamiado (séc. XV), Maria Adélia Soares de Carvalho Mendes (ed.). Porto: Fundação Eng. António de Almeida.

HUNT, T. (1997). Anglo-Norman Medicine: II. Shorter Treatises. Suffolk: Boydell \& Brewer.

IBN HABĪB (1992). Mujtasar fi l-tībb (Compendio de medicina), Camilo Álvarez de Morales y Fernando Girón Irueste (ed. y trad.). Madrid: CSIC-Instituto de Cooperación con el Mundo Árabe.

ISRAELI, I. (1945). Tratado de las fiebres, José Llamas (ed.). Madrid-Barcelona: Instituto «Arias Montano» de Estudios Hebraicos y Oriente Próximo.

- (1984). Tratado de las fiebres, Ruth M. Richards (ed.). Madison: Hispanic Seminary of Medieval Studies.

KETHAM, J. (1990). Compendio de la humana salud, M. ${ }^{a}$ Teresa Herrera (ed.). Madrid: Arco/Libros.

KINKADE, R. (1971). «Un nuevo manuscrito de la Semeiança del mundo». Hispanic Review 39-3, 261-262.

KÜHLEWEIN, H. (1884). «Beiträge zur Geschichte und Beurtheilung der kippokratischen Schriften». Philologus 42, 119-133.

KUHNE BRABANT, R. (1987-88). «The Arabic Prototype of the Capsula Eburnea». Quaderni di Studi Arabi 5, 431-441.

- (1989). «El Kitab al-dury, prototipo árabe de la Capsula Eburnea y representante más genuino de la tradición de los Secreta Hippocratis (I)». Al-qantara: Revista de Estudios Árabes 10, 3-20. 
KUHNE BRABANT, R. (1989). «El Kitab al-dury, prototipo árabe de la Capsula Eburnea y representante más genuino de la tradición de los Secreta Hippocratis (II)». Al-qantara: Revista de Estudios Árabes 10, 299-328.

- (1990). «El Kitab al-dury, prototipo árabe de la Capsula Eburnea y representante más genuino de la tradición de los Secreta Hippocratis (III)». Al-qantara: Revista de Estudios Árabes 11, 3-58.

- (1990b). «El tratadito pseudo-hipocrático Fî l-mawt al-sarî̀. Anaquel de Estudios Árabes 1, 237-262.

LENKIEWICZ, M. (1987). Contribución al estudio del léxico médico del español medieval: «Secretos de medicina» del licenciado don Juan Enriquez»y «Pronóstica del pseudo-Galeno» (Tesis de «Master of Arts»). Montreal: Department of Hispanic Studies, Mc Gill University.

LOBERA DE ÁVILA, L. (1542). Vergel de sanidad. Alcalá de Henares: casa de Joan de Brocar.

LÓPEZ FIGUEROA, L. (2011). Estudio y edición crítica de la compilación médica latina denominada «Tereoperica» (Tesis doctoral). Santiago de Compostela: Universidad de Santiago de Compostela.

MAESTRE GIL (1991). Libro de medecina llamado Macer, Thomas M. Capuano (ed.). Madison: Hispanic Seminary of Medieval Studies.

MENSCHING, G. (2002). «El Macer Floridus en la Península Ibérica: efectos y defectos secundarios de un herbario medieval». En Textos medievales y renacentistas de la Romania, M. ${ }^{\mathrm{a}}$ T. Navarro, J. Nitti y M. ${ }^{\mathrm{a}} \mathrm{N}$. Sánchez (eds.). Madison: Hispanic Seminary of Medieval Studies.

MOLINÉ I BRASÉS, E. (ed.) (1913). «Receptari de Micer Johan». Boletín de la Real Academia de Buenas Letras de Barcelona 7, 321-336 y 407-440.

MORAL DE CALATRAVA, P. (2012). «Frígidos y maleficiados. Las mujeres y los remedios contra la impotencia en la Edad Media». Asclepio. Revista de Historia de la Medicina y de la Ciencia 64-2, 353-372.

NUTTON, V. (1970). «Prognostica Galieni». Medical History 14, 96-100.

PENSADO FIGUEIRAS, J. (2012). «Pasajes del Macer Floridus castellano en el ms. II-3063 de la Real Biblioteca». Revista de Filología Española 92, 341-362.

- (2013). El códice Zabálburu de medicina medieval: edición crítica y estudio de fuentes (Tesis doctoral). A Coruña: Universidade da Coruña.

PEÑA, C. y GIRÓN, F. (2006). La prevención de la enfermedad en la España bajo medieval. Granada: Servicio de Publicaciones de la Universidad de Granada. 
PERARNAU I ESPELT, J. (1992). «El receptari del sabadellenc Joan Martina (1439)». Arxiu de Textos Catalans Antics 11, 289-328.

RAMELLO, L. (1994). «Una raccolta di ricette in antica lingua catalana (codice palatino 1052 della Biblioteca Nazionale di Firenze)». Quaderni di Filologia Romanza II, 99-136.

RENZI, S. (1852-1857). Collectio Salernitana (tomos I-V). Nápoles: Tipografia del Filiatre-Sebezio.

RIBELLES I COMÍN, J. (1915). Bibliografía de la lengua valenciana. Madrid: Tip. de la Rev. de Arch., Bibl. y Museos.

SUDHOFF, K (1915-1916). «Die hippokratische Krankheits-Prognostik nach dem Auftragen von Hautausschlagen Secreta Hippocratis oder Capsula eburnea gennant». Archiv für Geschichte der Medizin 9, 79-116.

TAAVITSAINEN, I. y PAHTA P. (eds.) (2004). Medical and Scientific Writing in Late Medieval English. Cambridge: Cambridge University Press.

VENTURA, I. (2011). «La medicina e la farmacopea della Scuola Medica Salernitana e le traduzione italiane: ipotesi di lavoro». En Volgarizzare, tradurre, interpretare nel secc. XIII-XIV, S. Lubello (ed.), 29-53. Strasbourg: ELIPHI, Éditions de Linguistique et de Philologie.

WENTZLAU, K. (1923). Frühmittelalteriliche und salernitanische Harntraktate. Eilenburg: F. Ernst Koch \& Sohn.

Recibido el 30 de junio de 2013.

Aceptado el 19 de septiembre de 2013. 